\title{
Phonation threshold pressure at large asymmetries of the vocal folds ${ }^{\star}$
}

\author{
Jorge C. Lucero \\ Dept. Computer Science, University of Brasilia, Brasília DF 70910-900, Brazil \\ Xavier Pelorson, Annemie Van Hirtum \\ LEGI, UMR CNRS 5519, Grenoble Alpes University, Saint-Martin-d'Hères, 38400 France
}

\begin{abstract}
The phonation threshold value of the lung pressure has been interpreted as a measure of ease of phonation and proposed as a diagnostic parameter for vocal health. Therefore, it is important to understand its behavior as a function of laryngeal parameters, particularly in abnormal configurations. This paper compares results from a theoretical model of the vocal folds with measures from a mechanical replica, in the presence of a natural frequency asymmetry. It shows that, at small asymmetry, the threshold pressure increases with the degree of asymmetry, whereas at large asymmetry, the threshold pressure reaches a plateau.
\end{abstract}

Keywords: phonation, vocal fold, oscillation, asymmetry 2010 MSC: 92C05, 74F10, 34C15

\section{Introduction}

During phonation, the right and left vocal folds act as a pair of coupled oscillators. In normal healthy conditions, they have a symmetrical configuration and oscillate in complete in-phase synchrony [1]. Abnormal conditions such as 5 tissue lesions and neurological disorders introduce right-left asymmetries which may hamper the oscillation and cause phase differences, complex entrainment regimes and other nonlinear phenomena [2, 3, 4, 5].

An important parameter of the vocal fold oscillation is the so-called phonation threshold pressure, which is defined as the minimum lung pressure required

\footnotetext{
*Presented in partial form at MAVEBA 2019: 11th International Workshop on Models and Analysis of Vocal Emissions for Biomedical Applications (Florence, Italy, December 17-19, 2019).

Email addresses: lucero@unb.br (Jorge C. Lucero), xavier.pelorson@univ-grenoble-alpes.fr (Xavier Pelorson), annemie.vanhirtum@univ-grenoble-alpes.fr (Annemie Van Hirtum)
} 
to start phonation [6]. The threshold pressure appears as a relevant parameter in equations of voice aerodynamics and has been interpreted as a measure of "ease of phonation" 7]. A number of studies have shown that its value increases with abnormal laryngeal conditions such as dehydration 8], fatigue 9, tissue scarring [10] and others, favoring its application as an assessment tool for vocal 15 health.

In a recent theoretical study, the effect of a natural frequency asymmetry on the threshold pressure was analyzed and different patterns at small vs. large asymmetries were detected [11]. If the asymmetry is small, then the threshold value pressure has a minimum at the symmetric configuration and increases 20 with the degree of asymmetry. On the other hand, if the asymmetry is large, then the threshold pressure assumes a constant value. The existence of such a region with constant threshold pressure would indicate caution in its proposed application for clinical diagnosis, because an increase or decrease of asymmetry (i.e., the abnormality) would not necessarily reflect in a respective increase or 25 decrease of threshold pressure, when the asymmetry is large. Thus, the present study has the purpose of analyzing further this effect by comparing the theory with data collected from a mechanical replica of the vocal folds.

\section{Theoretical model}

\subsection{Equations of motion}

Following [12], the right vocal fold is represented as a one-degree-of-freedom oscillator of the form

$$
M \ddot{x}_{r}+B\left(1+\eta x_{r}^{2}\right) \dot{x}_{r}+K x_{r}=P_{g},
$$

30 where $x_{r}$ is the tissue displacement, $M, B$ and $K$ are the mass, damping and stiffness, respectively, per unit area of the vocal fold medial surface, $\eta$ is a nonlinear coefficient to account for energy dissipation at large amplitudes, and $P_{g}$ is the glottal air pressure averaged over the medial fold surface. A similar equation in the variable $x_{\ell}$ is used for the left vocal fold. Both $x_{r}$ and $x_{\ell}$ are

35 defined as positive in the direction of glottal opening.

The glottal airflow is assumed frictionless, stationary, and incompressible, and any effects of the sub- and supraglottal systems are neglected. Then, the subglottal pressure is equal to the lung pressure, and the pressure at the glottal exit is atmospheric. Under such conditions, the glottal pressure $P_{g}$ may be approximated by

$$
P_{g}=\frac{\tau P_{s}}{k_{t} x_{0}}\left(\dot{x}_{r}+\dot{x}_{\ell}\right),
$$

where $\tau$ is the time delay for the superficial mucosal wave on the fold tissue to travel half the glottal depth (in the direction of the air flow), $P_{s}$ is the subglottal pressure, $k_{t}$ is a transglottal pressure loss coefficient, and $x_{0}$ is the glottal halfwidth (in the lateral direction) at the prephonatory position (phonation neutral 40 position) [11. 
The natural frequency of the right vocal fold is $\omega_{r}=\sqrt{K / M}$. Introducing a coefficient of asymmetry $Q$ for the natural frequency of the left vocal fold, in the form $\omega_{\ell}=Q \omega_{r}$ [13], then the system of equations for the right and left folds may be written as

$$
\begin{aligned}
& \ddot{x}_{r}+\beta\left(1+\eta x_{r}^{2}\right) \dot{x}_{r}+\omega_{r}^{2} x_{r}=\alpha\left(\dot{x}_{r}+\dot{x}_{\ell}\right), \\
& \ddot{x}_{\ell}+\beta\left(1+\eta x_{\ell}^{2}\right) \dot{x}_{\ell}+Q^{2} \omega_{r}^{2} x_{\ell}=\alpha\left(\dot{x}_{r}+\dot{x}_{\ell}\right),
\end{aligned}
$$

where $\beta=B / M$, and

$$
\alpha=\frac{S P_{s}}{k_{t} a_{0} c M}
$$

is the aerodynamic coupling, where $a_{0}=2 L x_{0}$ is the prephonatory glottal area, $L$ is the glottal length (in the anterior-posterior direction), $c=T /(2 \tau)$ is the mucosal wave velocity, $T$ is the glottal depth, and $S=L T$ is the medial surface of the vocal folds.

\subsection{Oscillation threshold}

The equilibrium position of the vocal folds is obtained by setting all derivatives to zero in Eqs. (3) and (4), which yields $x_{r}=0, x_{\ell}=0$. A standard stability analysis for that position produces the characteristic equation

$$
\begin{aligned}
s^{4}+2(\beta-\alpha) s^{3}+\left[\omega_{r}^{2}\left(Q^{2}+1\right)+\beta(\beta-2 \alpha)\right] s^{2} & \\
& +\omega_{r}^{2}\left(Q^{2}+1\right)(\beta-\alpha) s+Q^{2} \omega_{r}^{4}=0,
\end{aligned}
$$

where $s$ is a complex variable. When the subglottal pressure is $P_{s}=0$, then $\alpha=0$ and all roots of Eq. (6) have negative real parts [11]. In this case, the equilibrium position is stable. As $P_{s}$ increases, the roots cross the imaginary axis and the equilibrium becomes unstable. The oscillation threshold is obtained by letting $s= \pm i \omega$ and separating real and imaginary parts, which yields

$$
\begin{aligned}
& \omega^{4}-\left[\omega_{r}^{2}\left(Q^{2}+1\right)+\beta(\beta-2 \alpha)\right] \omega^{2}+Q^{2} \omega_{r}^{4}=0, \\
& (\beta-\alpha)\left[2 \omega^{2}-\omega_{r}^{2}\left(Q^{2}+1\right)\right]=0 .
\end{aligned}
$$

Solutions to Eq. 8 are

$$
\omega=\omega_{r} \sqrt{\frac{Q^{2}+1}{2}},
$$

and

$$
\alpha=\beta
$$

Substituting Eq. (9) in Eq. (7) produces

$$
\alpha=\frac{\beta}{2}+\frac{\omega_{r}^{2}}{4 \beta} \frac{\left(Q^{2}-1\right)^{2}}{Q^{2}+1} .
$$

Eq. [11 may be simplified by substituting $\omega_{r}$ from Eq. (9), and next defining

$$
\Delta=\frac{Q^{2}-1}{Q^{2}+1}
$$


which is a normalized asymmetry coefficient that maps $Q \in[0, \infty)$ into $\Delta \in$ $[-1,+1)$. Finally, substituting $\alpha$ from Eq. (5) and solving for the subglottal pressure, we obtain the oscillation threshold pressure

$$
P_{s}=P_{0}\left[1+\left(\frac{\omega \Delta}{\beta}\right)^{2}\right],
$$

where

$$
P_{0}=\frac{k_{t} \beta a_{0} c M}{2 S}
$$

is its value at the symmetric condition $\Delta=0$.

Another solution to Eq. (8) is given by Eq. 10, which produces the threshold pressure

$$
P_{s}=2 P_{0} \text {. }
$$

In this case, the oscillation frequency at the threshold is obtained by substituting Eq. 10 into Eq. 7], which yields

$$
\omega^{4}-\left[\omega_{r}^{2}\left(Q^{2}+1\right)-\beta^{2}\right] \omega^{2}+Q^{2} \omega_{r}^{4}=0
$$

and may have up to two real solutions.

Both Eqs. 13 and (15) coincide when

$$
|\Delta|=\beta / \omega
$$

The above results are summarized and illustrated in Fig. 1. At small asymmetries $(|\Delta| \leq \beta / \omega)$, the threshold pressure has a minimum at the symmetric configuration $(\Delta=0)$ and increases monotonically with the asymmetry following Eq. 13). The same pattern applies to the oscillation frequency. Both left and right vocal folds oscillate with the same frequency, given by Eq. 90. On the other hand, at large asymmetries $(|\Delta| \geq \beta / \omega)$, the threshold pressure assumes a constant value in relation to the asymmetry, given by Eq. (15). Two different

55 values of the oscillation frequency appear, given by Eq. 16); the lowest one corresponds to the lax vocal fold and the highest one to the stiff vocal fold. Thus, the vocal folds oscillate without synchronization, each one at its own oscillation frequency.

\section{Data collection}

Measures of oscillation threshold parameters were collected from a mechanical replica of the vocal folds. The replica and data collection method have been described in detail elsewhere [14, 15]. Briefly, the replica consists of two parallel latex tubes filled with water under pressure and supported by a metallic structure. The tubes represent the vocal folds in a 3:1 scale, and the internal water pressure of each tube can be set independently so that a stiffness asymmetry (and consequently, a natural frequency asymmetry) can be imposed in a controlled way. Air from a pressure reservoir is blown through a third latex tube, 

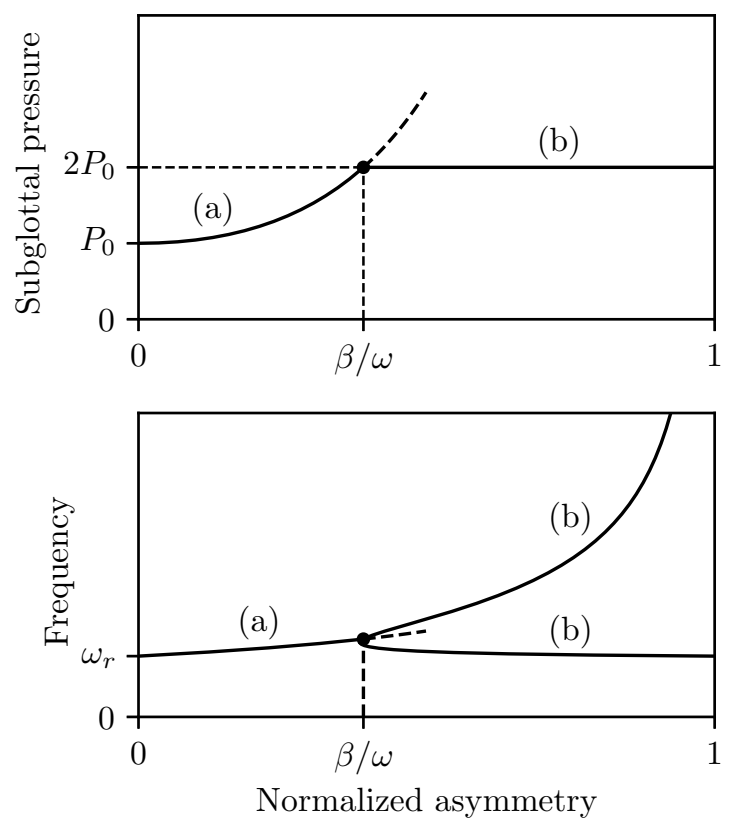

Figure 1: Oscillation threshold parameters. Top: subglottal pressure, (a) Eq. [13), (b) Eq. 15. Bottom: oscillation frequency, (a) Eq. (9), (b) Eq. 16. 

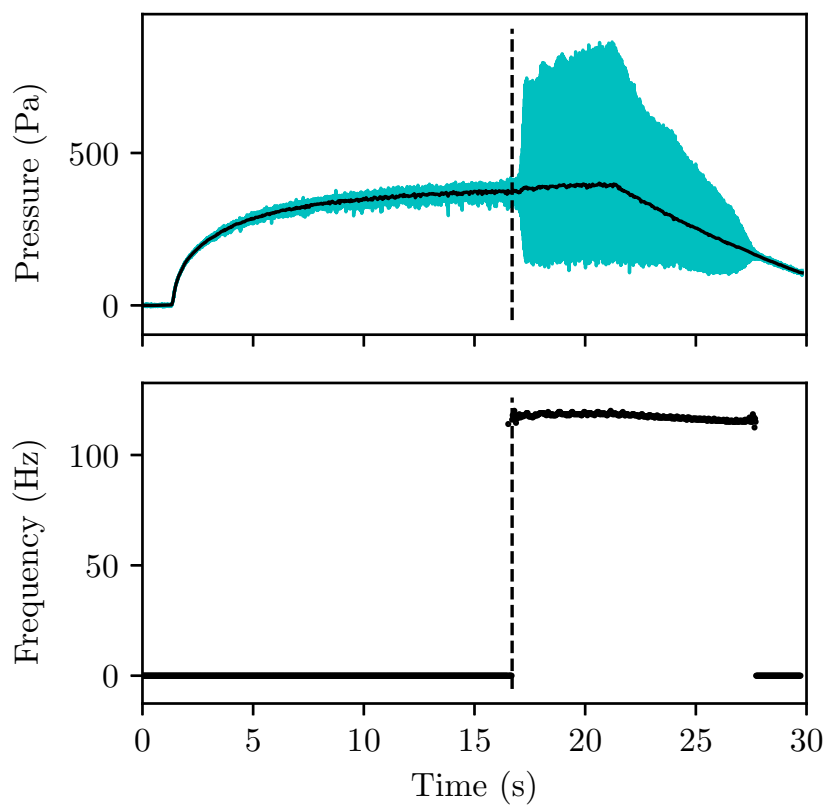

Figure 2: Example of the data collected from the replica when varying the upstream pressure. Top: subglottal pressure; the black curve is the mean pressure. Bottom: fundamental frequency. The broken line in each plot marks the oscillation threshold.

representing the glottal passage, situated in-between the two vocal fold tubes and perpendicular to them.

Values of oscillation threshold pressure and frequency were obtained by increasing the air pressure upstream of the replica from zero until an oscillation of the tubes was detected. The time instant of oscillation onset was determined by spectral analysis of the pressure signal measured immediately upstream the replica (subglottal pressure). Then, the mean subglottal pressure and oscil-

75 lation frequency at that instant were computed (Fig. 2). The glottal area at rest was determined from pictures taken by a digital camera, calibrated with a benchmark grid.

Three experiments were performed, in which measures of the above parameters (pressure, frequency and area) were taken at various values of internal

80 (water) pressures of the vocal fold tubes, in symmetrical and asymmetrical configurations. The theoretical model was next fitted to the collected data with the results shown in the next sections. 


\section{Experiment \#1}

In this experiment, the internal pressure of both vocal fold tubes was varied ${ }_{85}$ simultaneously between $4 \mathrm{kPa}$ and $8.5 \mathrm{kPa}$, keeping a symmetrical configuration. In the symmetric case, the theoretical model produces a threshold pressure $P_{s}=P_{0}$ and an oscillation frequency $\omega=\omega_{r}$.

The natural frequencies $\omega_{r}$ of the tubes were approximated by their resonance frequencies, obtained from [15]. The first (lowest) resonance frequency

90 is close to the oscillation frequency, and both are shown in the middle panel of Fig. 3 for comparison.

The threshold pressure was computed using Eq. (14). However, as the internal pressure of the tubes is increased, their volume also increases and all parameters in that equation vary. For the transglottal coefficient $\left(k_{t}\right)$ we adopted an expression of the form

$$
k_{t}=\frac{E}{a_{0}}+F,
$$

where $E$ and $F$ are coefficients to be determined [16]. The prephonatory glottal area $a_{0}$ was directly measured from the replica, as explained in the previous section, and is shown in the bottom panel of Fig. 3 The other parameters in Eq. (14) are more difficult to model or measure, and were simply lumped into a single coefficient, as follows. Substituting Eq. 18 into Eq. (14), we obtain

$$
P_{0}=G\left(1+F^{\prime} a_{0}\right),
$$

where $F^{\prime}=F / E$ and $G=\beta c M E /(2 S)$.

The top panel in Fig. 3 shows measures of threshold pressure and the fit provided by the above Eq. 19 , with $G=626.5 \mathrm{~Pa}$ and $F^{\prime}=-0.052 \mathrm{~mm}^{-2}$.

${ }_{95}$ The theoretical model produces a good prediction of the data, and we note that the increase of threshold pressure with internal pressure is a direct consequence of the glottal area variation.

\section{Experiment \#2}

In this experiment, the internal pressure of one fold was fixed at $6.0 \mathrm{kPa}$ whereas the internal pressure of the other was varied between $4.1 \mathrm{kPa}$ and 8.3 $\mathrm{kPa}$. The intention was to obtain both negative and positive values of the asymmetry coefficient $\Delta$, and the results are shown in Fig. 4

The oscillation frequency is well approximated by Eq. (9) (Fig. 4, middle panel). In this case, $\omega_{r}$ was set equal to the second resonance frequency of the 105 tubes, which was obtained from [15].

The threshold pressure was fitted using Eq. 13, and using the same approximation for $P_{0}$ as in Experiment \#1, which produces

$$
P_{s}=G\left(1+F^{\prime} a_{0}\right)\left[1+\left(\frac{\omega \Delta}{\beta}\right)^{2}\right] .
$$



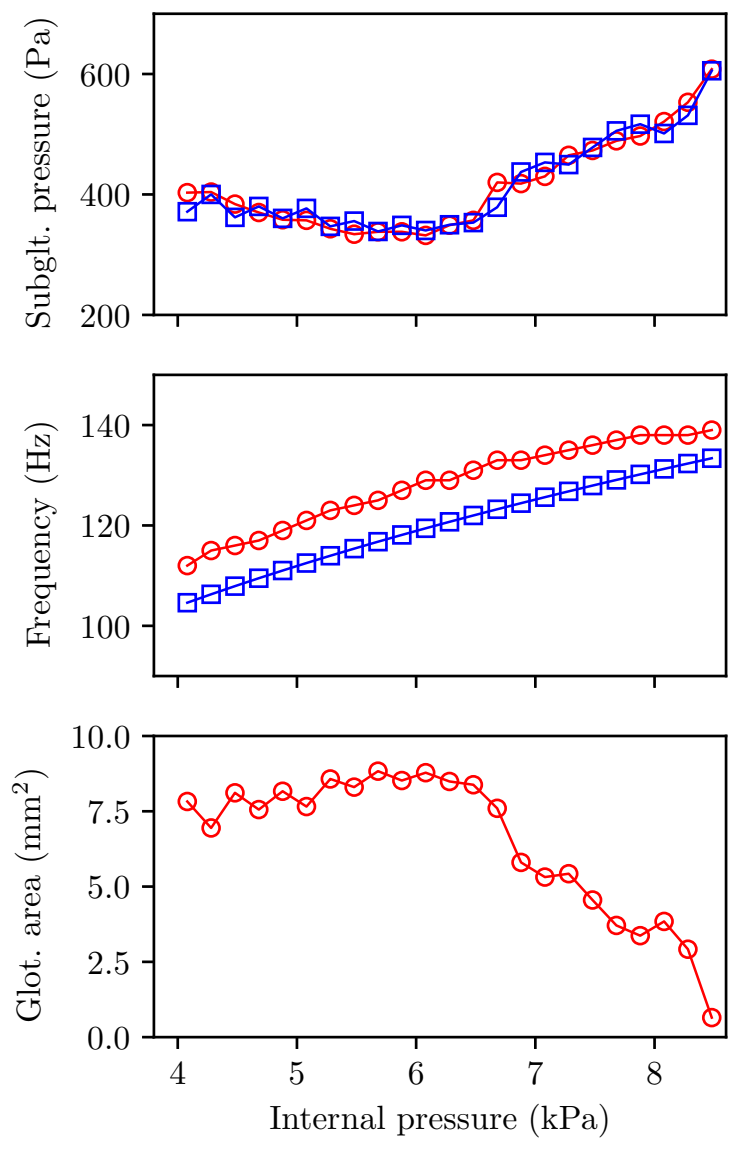

Figure 3: Results of Experiment \#1. Top: oscillation threshold pressure, middle: oscillation frequency, bottom: glottal area at rest. Red circles: collected data, blue squares: theoretical results. 
The top panel of Fig. 4 shows both the data and results from the above equation with $G=640.1 \mathrm{~Pa}, F^{\prime}=-0.039 \mathrm{~mm}^{-2}$, and $\beta=12900 \mathrm{~s}^{-1}$. Again, we obtained a good match between theoretical results and data.

The values of $|\Delta|$ that separate the regions of small and large asymmetry, given by Eq. (17), fall between 0.106 and 0.116 (depending on the oscillation frequency $\omega$ ). On the other hand, the range of values of $\Delta$ in this experiment is $[-0.116,0.099]$. Thus, the results are contained within the small asymmetry region (except extreme points at most), which justifies the selection of Eqs. 90 and 13 to fit the data.

\section{Experiment \#3}

In this experiment, the internal pressure of one fold was fixed at $2.5 \mathrm{kPa}$ whereas the internal pressure of the other was varied between $2.5 \mathrm{kPa}$ and 5.4 $\mathrm{kPa}$. The intention was to obtain larger values of the asymmetry coefficient $\Delta$, and the results are shown in Fig. 5 .

The top panel of Fig. 5 shows that the oscillation threshold pressure increases briefly for small values of $\Delta$, next reaches a plateau followed by a decrease. This pattern matches approximately the pattern in the top panel of Fig. 1] except for the decrease of pressure at the right. The border between the regions of small and large asymmetry would be then located at the point when the pressure reaches a plateau (approximately third data point), and the parameters of the theoretical model were adjusted to obtain such result.

The oscillation frequency was approximated by Eqs. (9) and (16) (Fig. 4 middle panel). The value of $\omega_{r}$ was set equal to the first resonance frequency of the tubes, as in Experiment \#1. Note that the theoretical model predicts two oscillation frequencies in the region of large asymmetry (one for each vocal fold oscillator, as explained in Section 2.2. . On the other hand, the experimental setup produces a single value of the oscillation frequency, which is closer to the highest of the theoretical frequencies. A possible interpretation is that the stiffer vocal fold tube dominates the oscillation entraining the lax one, in a phenomenon similar to the broadband synchronization detected in [11. However, such a phenomenon can not be captured by our present model owing to its simplicity.

The threshold pressure was fitted using Eqs. (13) and (15), and using the same approximation for $P_{0}$ as in Experiment \#1. The top panel of Fig. 5 shows both the data and results with $G=81.8 \mathrm{~Pa}, F^{\prime}=0.044 \mathrm{~mm}^{-2}$, and $\beta=27.9$ $\mathrm{s}^{-1}$. The fit is close for the small asymmetry and the plateau region, but it is not able to capture well the decrease of pressure in the right half of the plot. Note that the value of $\beta$ is much smaller from the one obtained for Experiment $\# 2$. One possible explanation could be that the replica was set in a different configuration, as shown by the larger values of prephonatory glottal area in the bottom panel of Fig. 5 .

The values of $\Delta$ that separates the regions of small and large asymmetry, given by Eq. (17), fall between 0.0477 and 0.0460 , approximately at the left of 

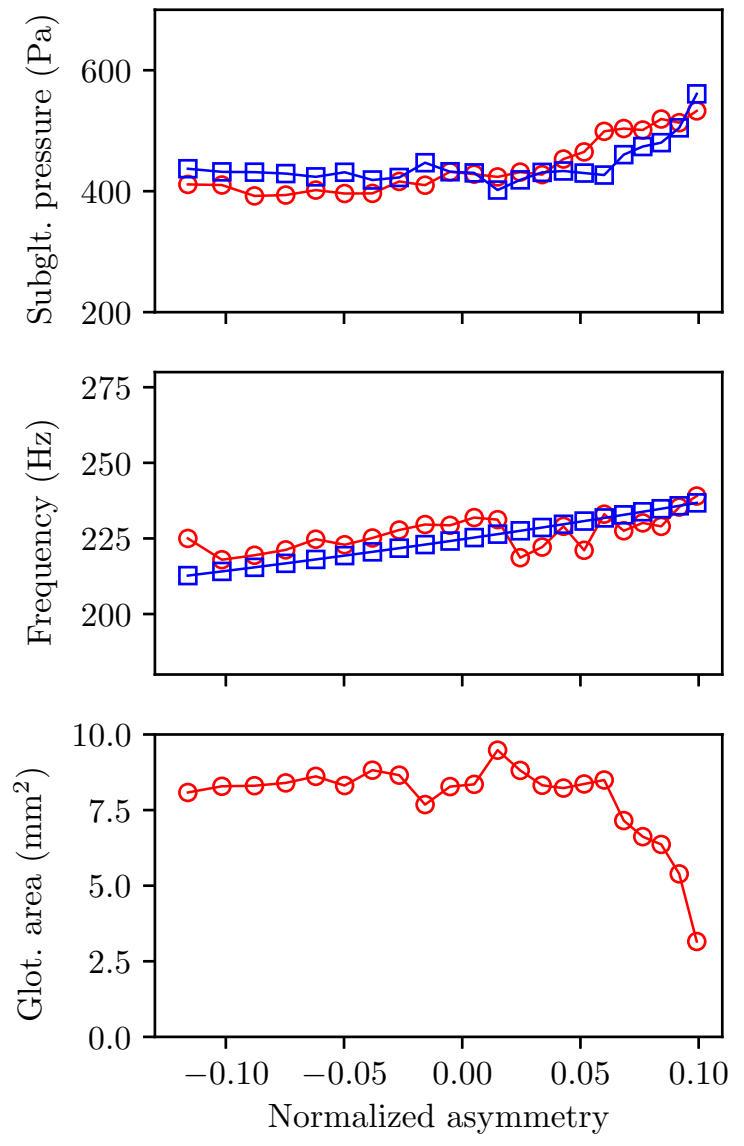

Figure 4: Results of Experiment \#2. Top: oscillation threshold pressure, middle: oscillation frequency, bottom: glottal area at rest. Red circles: collected data, blue squares: theoretical results. 
third data point (with $\Delta=0.0496$ ). The difference with the value obtained for

Experiment \#2 is caused by the smaller value of parameter $\beta$.

\section{Conclusions}

Our results show different patterns of variation for the phonation threshold pressure in regions of low vs. high asymmetry of the vocal folds. At low asymmetry, the threshold pressure increases with the asymmetry, whereas at large asymmetry the threshold pressure assumes a constant value or even a decrease, as shown by the measured data.

In general, the experimental models given by Eqs. 19 and (20) show good agreement with the collected data, except for the decrease of threshold pressure seen at large asymmetry, in Experiment \#3. A difficulty when comparing the models with the measured data is that variations of the internal pressure of the latex tubes affect not only their natural frequency but also their volume, introducing variations in parameters such as oscillating mass and medial surface that are not contemplated by the theory. Future research efforts will be dedicated to solve such difficulties with improved theoretical models and new experimental setups.

\section{Acknowledgments}

This work was done while Jorge C. Lucero was a visiting researcher of Grenoble-Alpes University at LEGI/CNRS UMR 5519 (Campagne chercheurs invités 2019 de l'Université Grenoble-Alpes). Jorge C. Lucero was also supported by CNPq (Brazil).

\section{References}

[1] I. Titze, Principles of voice production, Prentice Hall, Englewood Cliffs, N.J, 1994.

[2] U. Eysholdt, F. Rosanowski, U. Hoppe, Vocal fold vibration irregularities caused by different types of laryngeal asymmetry, European

Archives of Otorhinolaryngology 260 (8) (2003) 412-417. doi:10.1007/ s00405-003-0606-y.

[3] P. Mergell, H. Herzel, I. R. Titze, Irregular vocal-fold vibration - high-speed observation and modeling, Journal of the Acoustical Society of America 108 (6) (2000) 2996-3002. doi:10.1121/1.1314398.

[4] M. Tigges, P. Mergell, H. Herzel, T. Wittenberg, U. Eysholdt, Observation and modelling of glottal biphonation, Acustica 83 (4) (1997) 707-714.

[5] Z. Zhang, T. H. Luu, Asymmetric vibration in a two-layer vocal fold model with left-right stiffness asymmetry: Experiment and simulation, The Journal of the Acoustical Society of America 132 (3) (2012) 1626-1635. doi:10.1121/1.4739437 

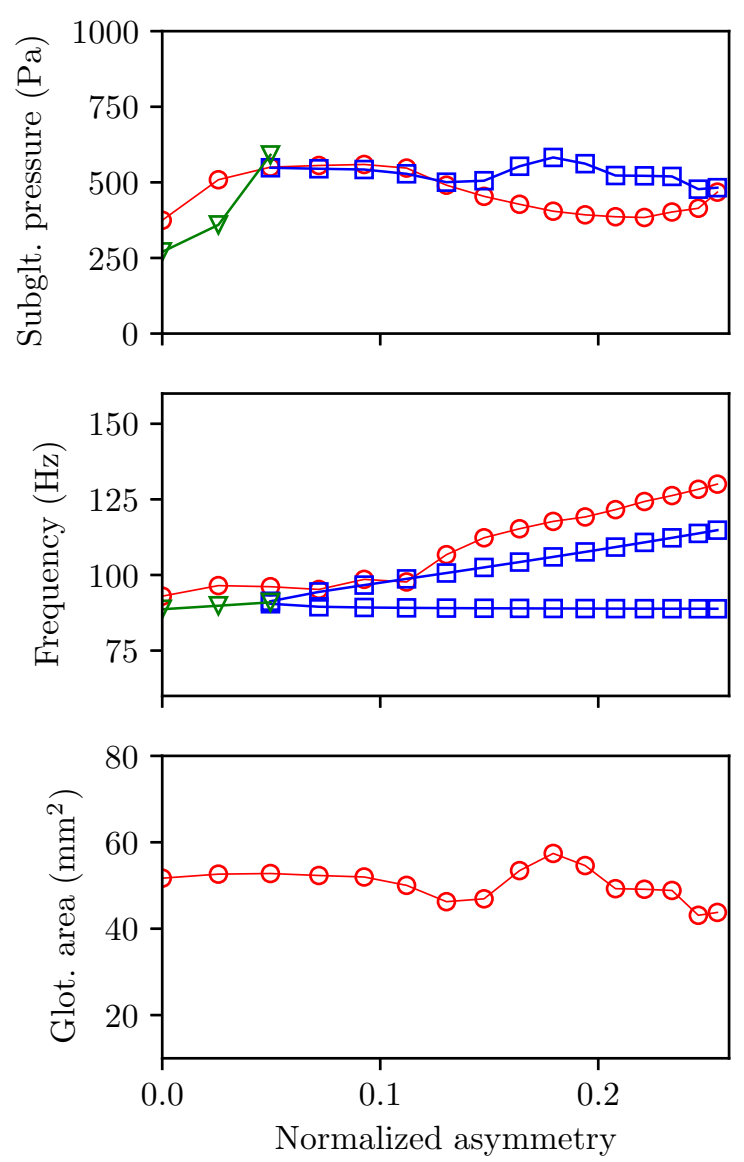

Figure 5: Results of Experiment \#3. Top: oscillation threshold pressure, middle: oscillation frequency, bottom: glottal area at rest. Red circles: collected data, blue squares: theoretical results for the large asymmetry region, green triangles: theoretical results for the small asymmetry region. 
[6] I. R. Titze, Phonation threshold pressure: A missing link in glottal aerodynamics, Journal of the Acoustical Society of America 91 (1992) 2926-2935. doi:10.1121/1.402928.

190 [7] I. R. Titze, S. S. Schmidt, M. R. Titze, Phonation threshold pressure in a physical model of the vocal fold mucosa, The Journal of the Acoustical Society of America 97 (5) (1995) 3080-3084. doi:10.1121/1.411870.

[8] K. Verdolini, Y. Min, I. R. Titze, J. Lemke, K. Brown, M. van Mersbergen, J. Jiang, K. Fisher, Biological mechanisms underlying voice changes due to dehydration, Journal of Speech, Language, and Hearing Research 45 (2) (2002) 268-281. doi:10.1044/1092-4388(2002/021)

[9] N. P. Solomon, M. S. DiMattia, Effects of a vocally fatiguing task and systemic hydration on phonation threshold pressure, Journal of Voice 14 (3) (2000) 341-362. doi:10.1016/s0892-1997(00)80080-6.

200 [10] S. Hirano, D. M. Bless, B. Rousseau, N. Welham, D. Montequin, R. W. Chan, C. N. Ford, Prevention of vocal fold scarring by topical injection of hepatocyte growth factor in a rabbit model, The Laryngoscope 114 (3) (2004) 548-556. doi:10.1097/00005537-200403000-00030.

[11] J. C. Lucero, J. Schoentgen, J. Haas, P. Luizard, X. Pelorson, Selfentrainment of the right and left vocal fold oscillators, The Journal of the Acoustical Society of America 137 (4) (2015) 2036-2046. doi:10.1121/1. 4916601 .

[12] R. Laje, T. Gardner, G. B. Mindlin, Continuous model for vocal fold oscillations to study the effect of feedback, Physical Review E 64 (5) (2001) 056201. doi:10.1103/physreve.64.056201.

[13] I. Steinecke, H. Herzel, Bifurcations in an asymmetric vocal-fold model, The Journal of the Acoustical Society of America 97 (3) (1995) 1874-1884. doi:10.1121/1.412061.

[14] J. Haas, P. Luizard, X. Pelorson, J. C. Lucero, Study of the effect of a moderate asymmetry on a replica of the vocal folds, Acta Acustica united with Acustica 102 (2) (2016) 230-239. doi:10.3813/aaa.918939.

[15] P. Luizard, X. Pelorson, Threshold of oscillation of a vocal fold replica with unilateral surface growths, The Journal of the Acoustical Society of America 141 (5) (2017) 3050-3058. doi:10.1121/1.4979935

[16] L. P. Fulcher, R. C. Scherer, Phonation threshold pressure: Comparison of calculations and measurements taken with physical models of the vocal fold mucosa, The Journal of the Acoustical Society of America 130 (3) (2011) 1597-1605. doi:10.1121/1.3605672 